EOMmUn: Communication et organisation

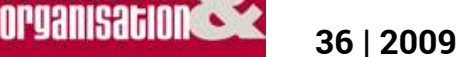

Pour une approche communicationnelle de l'individu au travail

\title{
De la rumeur à la peur
}

Une enquête de psychodynamique du travail à la suite d'un suicide lié au travail

\section{Pascale Molinier}

\section{OpenEdition}

\section{Journals}

Édition électronique

URL : http://journals.openedition.org/communicationorganisation/901

DOI : 10.4000/communicationorganisation. 901

ISSN : $1775-3546$

Éditeur

Presses universitaires de Bordeaux

Édition imprimée

Date de publication : 1 décembre 2009

Pagination : 44-54

ISBN : 978-2-86781-719-9

ISSN : $1168-5549$

Référence électronique

Pascale Molinier, «De la rumeur à la peur », Communication et organisation [En ligne], 36 | 2009, mis en ligne le 11 mars 2011, consulté le 19 avril 2019. URL : http://journals.openedition.org/ communicationorganisation/901 ; DOI : 10.4000/communicationorganisation.901 
Dossier : Pour une approche communicationnelle de l'individu au travail

\title{
Résumé
}

L'article s'appuie sur une enquête en psychodynamique du travail réalisée à la suite du suicide d'un jeune cadre auprès des personnels de son service. Le concept de "stratégie collective de défense » est utilisé pour rendre compte des distorsions de la communication entre les membres des équipes. L'intervention a mis en évidence une dégradation ancienne de la communication sur le travail, isolant chacun dans son rapport aux risques. Elle a permis d'analyser la peur masquée derrière l'expression des rumeurs et de mettre au jour leur dimension défensive.

\section{Mots-clefs}

Suicide, psychodynamique du travail, stratégies collectives de défense, peur, rumeur

\begin{abstract}
The article is based on an investigation involving work psychodynamics. It was carried out by interviewing staff following the suicide of a junior executive. The concept of "collective strategy of defence" is used to account for the distortion of the communication between the different members of the teams. The study revealed a long-standing lack of communication about work, each member of staff becoming isolated in their relationship to risk. It revealed the fear masked behind the rumours which were in turn shown to have a defensive dimension. By being disconnected the constraints of the organization of the work, the rumours contributed to deny them.
\end{abstract}

Keywords

Suicide, psychodynamics of work, collective strategies of defence, fear, rumour

Pascale Molinier, docteur en psychologie, HDR, est maître de conférences au CNAM - Centre de Recherches sur le Travail et le Développement (CRTD), CNAM, Paris. Ses recherches portent sur l'analyse du rapport entre travail et santé mentale, plus particulièrement dans le domaine des services aux personnes. Elle est l'auteur des enjeux psychiques du travail, Payot, 2006 et de Qu'est-ce que le care? (Molinier, Laugier, Paperman, ed.), Payot 2009. 


\section{De la rumeur à la peur. Une enquête de psychodynamique du travail à la suite d'un suicide lié au travail Pascale Molinier}

pascale.molinier@cnam.fr

Un jeune cadre, futur chef de son service, s'enferme dans son bureau pour mettre fin à ses jours avec des instruments de travail. Le drame intervient à peu de distance de la série de suicides très médiatisée parmi les salariés de grandes entreprises françaises en 2007. La question du statut du travail dans ce geste désespéré est immédiatement posée par certains membres du service. L'encadrement les accuse de vouloir «instrumentaliser» cette mort au profit de revendications portant sur les conditions de travail, tandis que se propagent des rumeurs concernant la vie privée du défunt ainsi que des pratiques de dissimulation attribuées à l'encadrement et à la direction. Il est fait état, en particulier, de plusieurs lettres du jeune homme, dont une adressée à ses collègues dont ceux-ci n'auraient pas eu connaissance. Quelques semaines plus tard, la direction fait appel à des chercheurs en psychodynamique du travail ${ }^{19}$, spécialisés dans le champ de la santé mentale au travail, pour répondre à la demande des équipes de les aider à s'extraire d'un état de sidération qui fait dire au membre le plus haut placé de l'encadrement: «depuis, tout continue, mais c'est comme si tout s'était arrêté ». Cette personne déplore aussi les rumeurs qui «salissent la mémoire » du mort. La rumeur s'entend donc ici comme une «infamie » (Froissart, 2002) qui briserait la réputation du jeune homme, en révèlerait des failles qui auraient dues rester du domaine privé, en même temps qu'elle opèrerait comme outil de manipulation en distillant la thèse du «travail qui tue ». Dans la phase de préparation de l'intervention, nous commençons par calmer les esprits : "non, nous n'allons pas chercher à prouver que le travail a "causé" le suicide de ce jeune homme». Nous allons plutôt essayer de comprendre quelles sont les incidences de cette mort tragique sur les autres membres des équipes et sur leur vécu au travail. Selon la méthodologie de la psychodynamique du travail (Dessors, 2009), l'analyse de la souffrance dans le travail implique, outre l'existence d'une demande émanant des salariés, la constitution de groupes de volontaires. L'élaboration collective du travail vise la

${ }^{19}$ L'enquête a été réalisée en 2008 avec Fabienne Benetti. 
Dossier : Pour une approche communicationnelle de l'individu au travail

socialisation des difficultés et le déplacement des défenses susceptibles de faire obstacle à la discussion. Notre dispositif d'enquête offrait un espace collectif de parole dont chacun savait qu'il autorisait explicitement l'expression de la fragilité psychologique aussi bien que la discussion sur les aléas du travail. La seule condition requise pour y participer était de penser qu'il était nécessaire de parler ensemble du drame et de ses conséquences pour chacun. Nous nous sommes réunis deux fois trois heures avec un groupe de quinze assistants techniques (les subordonnés directs du jeune cadre) et un autre de huit techniciennes (travaillant quotidiennement avec lui mais n'étant pas ses subordonnées). Aucun cadre n'a souhaité participer à un troisième groupe ${ }^{20}$. Ceci constitue une limite du travail qui a pu être accompli, comme cela a été souligné dans le rapport final restitué aux deux groupes puis à l'encadrement. Une dernière séance de discussion (trois mois plus tard) a réuni l'encadrement, les équipes et nous-mêmes. Les rumeurs et les diverses accusations portées par les uns contre les autres sont les manifestations bruyantes à travers lesquelles les collègues du jeune homme ont tout d'abord tenté de se protéger de la souffrance générée par sa mort tragique; en d'autres termes, ces distorsions de la communication relevaient d'une fonctionnalité avant tout défensive, que nous développons au cours de cet article.

\section{Des stratégies collectives de défense}

Travailler implique souvent de se confronter à des risques qui peuvent toucher à sa propre intégrité ou à celle des autres. Dans la situation présente, les équipes exercent un travail dangereux dont les risques génèrent de la peur pour l'encadrement comme pour les subordonnés. La psychodynamique du travail a montré que la peur empêche de travailler si elle n'est pas contenue par des stratégies collectives de défense (Dejours, 1993). Celles-ci visent à réduire la perception du danger en opérant dans un registre symbolique: le risque n'est pas transformé, il est collectivement impensé ; et la meilleure manière de ne pas y penser, c'est encore de ne pas en parler. Les stratégies de défense ont ainsi une incidence directe sur les formes de la communication interpersonnelle, celles-ci s'organisant de manière à

\footnotetext{
${ }^{20}$ L'absence des encadrants dans le dispositif d'enquête est liée, pensonsnous, à une censure exercée par le cadre de plus haut niveau. Sur le principe de la communication paradoxale, cette personne a rendu possible notre intervention en réalisant des prouesses organisationnelles pour libérer du temps collectif aux équipes, mais elle a réagi de façon agressive à l'évocation des imperfections organisationnelles indiquées dans le rapport d'enquête.
} 
occulter ce qui fait souffrir dans le travail. Quand les difficultés s'accumulent sans qu'il y soit remédié, les défenses collectives se radicalisent. Des pans très importants de la réalité du travail ne sont plus discutés, au point que l'on peut parler de «pathologies de la communication» (Dejours, 1993). Chacun est alors seul avec sa connaissance du réel qu'il ne peut plus mettre en partage avec autrui. Cette situation a été décrite sous les termes d'aliénation sociale (Sigaut, 1990) ou de pathologie de l'isolement (Dejours, 2007), tandis que la communication qui s'organise autour de l'évitement ou du déni du travail a été décrite sous celui d'aliénation culturelle (Sigaut, 1990).

Dans notre enquête, la circulation de la rumeur répond à ce type de fonctionnalité défensive : socialiser le drame tout en parlant d'autre chose que des risques du travail. Les assistants techniques ont développé des stratégies de défense viriles (Molinier, 2006). Exprimer sa vulnérabilité n'est pas de mise : le côté combatif et agressif est ici valorisé. Tous les participants s'accordent à décrire les relations entre assistants techniques et encadrants comme un bras de force épuisant. Cette armure virile est encore renforcée par l'activisme. La charge de travail va croissant, mais sous prétexte de ne pouvoir faire autrement, on «fonce » et l'on s'évite ainsi de mettre en discussion les aléas, les moments de peur ou d'incertitude. La mise à disposition de six heures de discussion pour notre enquête (qui a nécessité la fermeture du service) a ainsi constitué une rupture notable avec le mode de fonctionnement habituel des équipes. Cependant, cette tendance globale à «la fuite en avant » montrait déjà quelques signes de rupture avant le décès du jeune cadre. C'est du moins ce que suggèrent les propos de certains assistants techniques. Ils espéraient que leur futur chef résoudrait un certain nombre de dysfonctionnements dont l'actuel encadrement ne voulait pas entendre parler ${ }^{21}$ : «On lui avait montré tout ce qui ne marche pas, on a dû lui faire peur ». Précisément, le premier constat de notre enquête a été l'envahissement de la vie psychique des participants par une peur qui ne pouvait plus être écartée, ni cependant se partager spontanément; son expression demeurant interdite par les règles défensives qui bornaient préalablement les limites de la communication. Dans la première

${ }^{21}$ À l'évidence, le cadre supérieur n'était pas exempt de peur (ou de culpabilité), mais il lui était impossible d'en parler (même avec nous qui l'avons vu longuement à plusieurs reprises, avec un assez bon contact). 
Dossier : Pour une approche communicationnelle de l'individu au travail

séance du groupe des assistants techniques, la peur s'est d'abord exprimée sous une forme particulièrement angoissante: celle du fantôme du mort. Plusieurs personnes ont cru l'apercevoir au bout d'un couloir, au fond d'un local retiré ou désert : "j'ai eu peur, j'ai fait demi-tour ». Certains se sont sentis «poursuivis» par lui ou l'ont vu apparaître dans leur chambre à leur réveil, "j'ai vu sa tête dans une bulle». D'autres encore, au moment de réaliser certains gestes professionnels, ont été pétrifiés par la pensée qu'il avait fait ce geste pour se tuer. Toutes ces «apparitions » avaient un sens lié à la peur générée par l'activité comme travailler seul dans des lieux isolés et/ou réaliser des gestes dangereux. La possibilité d'oser livrer ces "pensées irrationnelles », de découvrir que d'autres avaient vécu des expériences similaires, a été un temps initial très fort où chacun a pu prendre la mesure de sa propre vulnérabilité et de celle des autres. Les participants ont aussi fait l'expérience que l'influence psychologique de ce drame ne se laissait pas dissocier de leur expérience ordinaire du travail. Cet évènement tragique rendait celle-ci insupportable et chacun ne reprendrait le cours normal des choses qu'à la condition de mettre à plat les problèmes posés par l'activité.

\section{Des sentiments peu conformes au respect du mort}

Des rumeurs, en effet, il en circulait beaucoup. Il se chuchotait qu'il existait des antécédents de suicide et de bizarreries chez les ascendants du défunt. On parlait d'une dispute avec sa femme, de gros problèmes d'argent. Certaines personnes ne pouvaient s'empêcher d'exprimer leur colère contre ce père de famille auquel ils ne "pardonnaient pas » d'abandonner plusieurs enfants et de les abandonner eux aussi, alors qu'ils comptaient sur lui. D'autres avaient ouï dire qu'il souhaitait démissionner et avait pris contact avec l'entreprise concurrente... D'autres bruits concernaient l'attitude de l'encadrement. Des assistants techniques s'insurgeaient, avec une angoisse palpable, contre le fait que les lettres retrouvées dans le bureau auraient été confisquées et qu'ils en ignoraient le contenu. "On a tourné la page un peu vite, comme si rien ne s'était passé » diront les participants se plaignant de n'avoir pas eu «le droit» d'en parler. La suite de la discussion a fait apparaittre que ce sentiment d'un interdit de «dire », d'abord attribué à l'encadrement de façon univoque, recouvrait aussi de la part des équipes des sentiments de culpabilité et de colère. Que signifiait, par exemple, l'insistance de certains à vouloir voir à tout prix les lettres de leurs propres yeux? Nous avons fini par comprendre, en recoupant leurs propos avec ceux de la direction, que leur contenu était en réalité connu de tous. Il n'y avait pas plusieurs lettres, mais une seule où le jeune homme s'accusait de «son 
incompétence personnelle et professionnelle ». Cette lettre n'était pas dans les mains de la direction, mais dans celles de son épouse. Si les assistants techniques n'arrivaient pas à croire ce qu'ils savaient pourtant, c'est qu'ils redoutaient qu'il y eut néanmoins «autre chose » qu'on leur eut caché. Plusieurs d'entre eux s'étaient affrontés durement avec le jeune cadre. « 48 heures avant [sa mort], je me suis accroché avec lui, je me suis senti un peu fossoyeur », dira l'un d'eux. Ce sentiment de culpabilité - de l'avoir souvent contredit de façon peu courtoise, d'être passé outre ses ordres ou d'avoir refusé de coopérer quand ce qui leur était demandé ne leur paraissait pas conforme du point de vue de la sécurité - était demeuré indicible, tout comme la persistance de leur ressentiment et de leur colère contre le jeune homme. La mort de celui-ci n'avait pas soldé le conflit. Les accusations de dissimulation de la part de la direction masquaient ainsi une angoisse - celle d'y être pour quelque chose - et une agressivité non tarie contre quelqu'un dont il était devenu cependant délicat de parler en mal. Rien n'est a priori irrecevable, indécent ou hors de propos quand il s'agit de comprendre ce qui fait souffrir les gens et comment ils s'en défendent. Nous avons toléré, dans le dispositif d'enquête, l'expression virulente des désaccords portant sur le travail avec le jeune cadre. Rapidement, les assistants techniques en sont arrivés à l'idée que ces désaccords ne concernaient pas seulement leur relation avec le défunt, mais qu'ils existaient aussi entre eux, bien qu'ils ne les mettent plus en débat. La rigidification croissante des procédures les rendait la plupart du temps inapplicables, contraignant chacun à des formes de contournement qui auraient pu exposer à des sanctions, mais sans lesquelles il était impossible de travailler. Le travail réel était pour cette raison en passe de devenir clandestin, ce qui majorait la peur, puisque chacun travaillait sans dire ce qu'il faisait «réellement», ni sans savoir ce que faisaient les autres. La discussion s'est alors organisée autour d'une tension entre d'un côté le respect des procédures comme nécessaires et protectrices et de l'autre, l'expérience que le travail réel nécessite de constants ajustements et de la souplesse. Montrer au jeune cadre ce qui «ne marchait pas» avait donc été une tentative pour essayer de le déplacer de sa posture prescriptive. Mais celui-ci était-il en mesure de trouver des appuis du côté de ses collègues pour assumer la connaissance du travail réel ?

\section{Fragilité structurelle et souffrance dans le travail}

On notera que, dans sa lettre, le jeune cadre s'accuse d'une «incompétence personnelle et professionnelle ». Bien que le débat public sur les suicides liés au travail se polarise aujourd'hui autour de deux thèses adverses, l'une reversant toute causalité au registre d'une 
Dossier : Pour une approche communicationnelle de l'individu au travail

fragilité personnelle, l'autre au registre d'une organisation du travail pathogène, nous savons de longue date que cette intrication malheureuse entre les deux sphères est de règle dans les décompensations psychopathologiques (Veil, 1954). Et si nous nous accordons avec la thèse de la centralité du travail dans le fonctionnement mental, alors, ainsi que le souligne Philippe Davezies, « suite à un suicide, il est au minimum légitime de s'interroger sur le fait que l'activité professionnelle et les relations de travail n'ont pas rempli leur fonction de protection de la santé » (Davezies, 2007 : 33). Ce qui est malheureusement le cas dans la situation présente.

À la thèse d'une «fragilité structurelle », au sens psychopathologique du terme, certains auteurs privilégient celle d'une forme particulière d'engagement dans le travail. Selon Christophe Dejours, «ceux qui se suicident au travail se recrutent parmi les hommes et les femmes qui se sont les plus engagés dans le travail, parmi les meilleurs» (Dejours, 2007 : 2). Mais qui décide de qui sont les «meilleurs »? Et selon quels critères de «compétence»? Dans notre enquête, chacun en fonction de son degré de proximité dans le travail, de son rapport de hiérarchique ou de subalterne, a sa propre version de l'engagement du défunt dans son travail, des difficultés qu'il y rencontrait ou qu'il faisait subir à d'autres. Chacun a aussi son interprétation des raisons qui l'ont conduit à revenir sur son lieu de travail pour mettre fin à ses jours. Pour les uns, c'est le signe d'une souffrance dans le travail. Ce sont ceux qui ont le sentiment de rencontrer des difficultés grandissantes dans l'exercice de leur métier; le geste suicidaire fait écho à leur propre souffrance. D'autres l'interprètent comme le signe d'une ambivalence, d'un suicide sur le mode «appel à l'aide », l'entreprise leur apparaissant comme un lieu peuplé où l'on peut encore espérer être sauvé, par différence avec la forêt voisine. Ils s'interrogent donc plutôt sur les conditions qui n'ont pas permis de porter assistance. Comment n'ont-ils pu se douter de rien ? Tous considèrent ce jeune cadre comme «brillant», mais certains jugent son savoir livresque, son rapport à la prescription rigide, quand d'autres pensent qu'il agissait avec une rigueur protectrice contre certaines dérives. Certains le décrivent peu ouvert au dialogue, clôturant les discussions de son autorité hiérarchique, d'autres pensent tout le contraire. Certains l'estiment sûr de lui, d'autres lui attribuent un sentiment d'insécurité qu'ils partagent ou craignent de lui avoir fait partager, comme nous l'avons déjà mentionné. Ce portrait à facettes multiples montre bien que le jugement sur le travail, sa qualité, le degré d'engagement qu'il implique, est variable en fonction des points de vue. Sont-ce les «meilleurs» qui se suicident ou ceux dont 
l'économie pulsionnelle trouve à s'investir - et à s'épuiser - dans l'idéologie « exaltante» de la performance et la concurrence ? Ceux qui ne peuvent adhérer aux stratégies collectives de défense ? Ou ceux qui ont le plus de difficultés à s'autoriser une souplesse par rapport à la prescription? Ceci déplace la question à la fois du côté des conditions collectives qui autorisent cette souplesse - et reboucle sur les pathologies de l'isolement analysées par Christophe Dejours - mais aussi du côté des configurations psychologiques individuelles. L'ingéniosité, la «mètis» (Detienne, Vernant, 1974), implique une certaine souplesse psychique, de pouvoir renoncer à des conceptions idéalisées du travail et de soi-même, donc un certain rapport au réel et à l'échec. Tout type de «bidouillage » et de modestie devant la tâche qui, dans cette entreprise, était rendu particulièrement difficile pour l'encadrement, du fait d'un ensemble de contraintes existant dans une activité à risques, mettant directement en cause sa responsabilité pénale en cas de problèmes, et du fait des défenses viriles des assistants techniques «rebelles», toujours promptes à ne pas obtempérer.

Celles que nous appelons les techniciennes, en revanche, pouvaient s'autoriser «d'arrondir les angles », mais au prix d'un certain mépris de collègues, les jugeant "effacées », soumises à la hiérarchie et moins directement indispensables ${ }^{22}$. Elles apparaîtront, à l'issue de l'enquête, et selon leurs propres termes, comme les «casques bleus » qui tentent de concilier et de réduire les affrontements entre les différentes logiques de métier. Un rôle médiateur et pacificateur dont elles ne peuvent s'affranchir du fait de leur travail de coordination et d'appui technique au service du travail des autres qui implique pour être bien fait (et pour sortir à l'heure) d'être en mesure de se mettre à leur place. Ce sont elles qui donneront la description la plus détendue du défunt, un «type chouette » qui s'attardait pour prendre le café et l'une d'entre elles qui repéra avec certitude que celui-ci était dans un état anormal d'excitation la veille de sa mort. Or, si après ce drame, quelque chose de l'espace de délibération sur le travail peut se

${ }^{22}$ Les assistants techniques doivent être détenteurs d'un diplôme précis, qualification relativement rare, ce n'est pas le cas des techniciennes recrutées à partir de formations diplômantes plus fréquentes. Les premiers ont donc un pouvoir dans le rapport de force avec la hiérarchie. Il est à noter qu'il y a des femmes parmi les assistants techniques et au moins un homme parmi les techniciens. La plupart des femmes (mais pas toutes) dans l'équipe des assistants partagent les défenses viriles de leurs collègues. Le technicien déploie les mêmes compétences d'attention aux autres que les techniciennes. 
Dossier : Pour une approche communicationnelle de l'individu au travail

recomposer, cela ne se fera pas sans mobiliser la pugnacité des assistants techniques, certes, mais aussi le travail attentionné des techniciennes, un travail qui est déjà là, mais confondu avec de la soumission. Pour le dire autrement: cela ne se fera pas sans modification des représentations de qui sont les «meilleurs» et les «moins bons» et donc sans une transformation des modalités de la reconnaissance du travail.

\section{Conclusion}

Cette enquête a mis en évidence une détérioration déjà ancienne de la discussion sur le travail au sein des équipes, isolant chacun dans son rapport aux risques croissants de l'activité. Elle a permis d'analyser la peur masquée derrière les rumeurs et de mettre au jour que les stratégies collectives de défense - virilité défensive et activisme - ne remplissaient plus leur fonction préservatrice de la santé mentale. Au contraire, celles-ci s'étaient radicalisées au point de faire obstacle à toute mise en discussion du travail réel ${ }^{23}$.

Par sa violence, son caractère inattendu et son impact affectif, le suicide du jeune cadre a révélé que le travail était devenu difficile pour tous, impliquant une reprise du débat sur les contraintes de l'organisation du travail. Avant notre intervention, en lieu et place d'une discussion sur le travail, s'était développée une personnalisation du drame et des positions de chacun sous la forme de rumeurs, d'accusations et de pathologies de la communication (Dejours, 1993). Ces distorsions n'ont pu être surmontées qu'à la condition d'un travail collectif d'explicitation et d'élaboration du vécu de ce drame, sans le dissocier de son contexte, c'est-à-dire de l'activité réalisée ensemble et des relations de travail entre tous et avec le défunt. Évoquer l'hypothèse d'une "fragilité structurelle» du jeune homme (ses «antécédents ») n'a pas empêché ses collègues de s'identifier à sa détresse, ni surtout de s'interroger sur leurs propres responsabilités dans le processus de déstructuration des liens collectifs. La restitution du rapport d'enquête en présence de l'encadrement a montré que même si celui-ci n'a pas «baissé la garde », continuant à mal tolérer les critiques adressées à l'organisation du travail, la qualité de la discussion menée durant l'enquête a permis que celle-ci se poursuive dans une tonalité moins «musclée » et plus respectueuse que celle qui prévalait avant l'enquête.

${ }^{23}$ Ce processus de radicalisation des défenses est connu sous le terme d'« idéologie défensive de métier » (Molinier, 2006) 
L'analyse psychodynamique de cette situation localisée ne permet pas la montée en généralité, mais elle suggère que le contenu de ce que dit la rumeur (les accusations de dissimulation à l'encontre de l'encadrement, les antécédents psychiatriques dans la famille du jeune cadre, ses problèmes d'ordre personnel) est parfois moins important que «ce qu'elle ne dit pas » et qu'elle contribue à masquer, c'est-àdire la peur des risques encourus dans le travail. Le problème n'est pas non plus celui de savoir si ce contenu est «vrai» ou «faux» (Campion-Vincent, Renard, 2002). En l'occurrence, la dissimulation de l'encadrement s'est avérée fausse et les antécédents psychiatriques, les problèmes personnels, conjugaux ou économiques, n'ont pas été prouvés. Ce qui importe ici est l'usage qui est fait de la rumeur comme mode de communication dans un contexte où les personnes doivent recomposer des défenses collectives contre la peur dans le travail, l'ancien système défensif ayant été déstabilisé par le drame du suicide. La compréhension des effets de la rumeur gagnerait peut-être, de façon plus générale, à être analysée aussi du point de vue de son éventuelle contribution à la construction collective d'un déni de réalité pour ne pas penser ce qui fait souffrir dans le travail, ou plus largement dans la société.

Dans la situation analysée ici, les rumeurs, comme formes de pathologies de la communication, sont le symptôme de l'absence de débat sur le travail. Plus largement, les connaissances en psychodynamique du travail suggèrent que la communication sur le travail ne se prescrit pas. Elle résiste aux injonctions même les mieux intentionnées et dépend entièrement de l'engagement volontaire des personnes dans un processus collectif où l'authenticité de la parole sur le travail est tributaire de l'espoir qu'il en sera tenu compte pour réduire les risques et la pénibilité. Lorsque l'information sur le travail n'est pas reliée à des conditions de publicité, comme dans le cas des techniciens disant «on lui a montré tout ce qui ne marchait pas, on a dû lui faire peur », l'information peut s'avérer anxiogène, voire plus déstabilisatrice encore. Il est potentiellement très délétère pour la santé mentale d'avoir connaissance, par exemple, du dysfonctionnement d'un système de barrières automatiques et de vivre, sans pouvoir le partager, avec la hantise qu'un train écrase une voiture. En ce sens, les interrogations et la culpabilité des assistants techniques ne sont pas complètement sans fondements. Au vu des éléments recueillis dans l'enquête, nous pouvons penser que la dégradation des formes de coopération et de vivre ensemble ont contribué à isoler ce futur chef de service face à de très fortes attentes des équipes et dans un contexte de sécurité particulièrement anxiogène parce que difficile à apprécier. 
Dossier : Pour une approche communicationnelle de l'individu au travail

Créer et entretenir un espace de discussion interne sur le travail implique une volonté collective, à tous les niveaux hiérarchiques, de mettre en débat le travail réel pour s'accorder ensemble sur les meilleurs compromis entre les différentes contraintes, ce qui constitue la meilleure prévention des formes de décompensations liées à la souffrance dans le travail.

\section{Bibliographie}

CAMPION-VINCENT V., RENARD J-B. De source sûre. Nouvelles rumeurs d'aujourd'hui, Paris : Payot, 2002.

DAVEZIES P. De mauvaises réponses à une vraie question. in : Santé \& Travail. $\mathrm{n}^{\circ} 60,2007$, pp. 29 -31, 2007.

DEJOURS C. Pathologie de la communication, situations de travail et espace public : le cas du nucléaire. Raisons Pratiques, 1993, 3, pp. 177-201.

DEJOURS C. Une nouvelle forme d'aliénation qui tue. in : Santé \& Travail, ${ }^{\circ} 60,2007$, pp. 2 - 28.

DETIENNE M., VERNANT J-P. Les ruses de l'intelligence. La mètis chez les Grecs. Paris : Flammarion, 1974.

DESSORS D. De l'ergonomie à la psychodynamique du travail. Méthodologies de l'action. Toulouse : Éditions Érès, 2009.

FROISSART P. La rumeur. Histoire et fantasmes. Paris : Belin, coll. « Débats », 2002.

MOLINIER P. Les enjeux psychiques du travail. Introduction à la psychodynamique du travail, Paris : Payot, 2006.

SIGAUT F. Folie, réel et technologie. in : Techniques et culture. 1990, 15, pp. 167-179. Réédité dans Travailler, 2004, 12, pp. 117-134.

VEIL C. Phénoménologie du travail. in : L'évolution psychiatrique, 1954, 4, pp. 693-721. Réédité dans Travailler, 1999, 2, pp. 13-38. 